\title{
Mitochondria-Specific Monoclonal Antibodies in Eggs and Embryos of the Ascidian Halocynthia roretzi
}

\author{
Yong Han Baek*, Wang Jong Lee* and †Gil Jung Kim \\ Dept. of Marine Molecular Bioscience, Gangneung-Wonju National University, \\ Gangneung 25457, Republic of Korea
}

\begin{abstract}
Ascidian embryos have become an important model for embryological studies, offering a simple example for mechanisms of cytoplasmic components segregation. It is a well-known example that the asymmetric segregation of mitochondria into muscle lineage cells occurs during ascidian embryogenesis. However, it is still unclear which signaling pathway is involved in this process. To obtain molecular markers for studying mechanisms involved in the asymmetric distribution of mitochondria, we have produced monoclonal antibodies, Mito-1, Mito-2 and Mito-3, that specifically recognize mitochondriarich cytoplasm in cells of the ascidian Halocynthia roretzi embryos. These antibodies stained cytoplasm like reticular structure in epidermis cells, except for nuclei, at the early tailbud stage. Similar pattern was observed in vital staining of mitochondria with $\mathrm{DiOC}_{2}$, a fluorescent probe of mitochondria. Immunostaining with these antibodies showed that mitochondria are evenly distributed in the animal hemisphere blastomeres at cleavage stages, whereas not in the vegetal hemisphere blastomeres. Mitochondria were transferred to the presumptive muscle and nerve cord lineage cells of the marginal zone in the vegetal hemisphere more than to the presumptive mesenchyme, notochord and endoderm lineage of the central zone. Therefore, it is suggested that these antibodies will be useful markers for studying mechanisms involved in the polarized distribution of mitochondria during ascidian embryogenesis.
\end{abstract}

Key words : Ascidian, Monoclonal antibody, Differential segregation and distribution of mitochondria

\section{INTRODUCTION}

Mitochondria are not only one of the major ancient endomembrane systems in eukaryotic cells but they are also the major energy generators in nearly all eukaryotic cells (Coffman \& Denegre, 2007; Friedman \& Nunnari, 2014). Mitochondrial numbers vary depending on the cell type and can change with the energy needs of the cell. In a liver cell, mitochondria are present about 1,000 to 2,000 (Alberts et al., 2013). Neural and muscle cells may contain many thousands of mitochondria. Mitochondria are seen to form complex branched networks inside the cell with cytoskeleton (Egner et al., 2002; Friedman \& Nunnari, 2014). The association with the cytoskeleton, such as microtubule and vimentin, regulates the distribution, function and shape of mitochondria (Hoitzing et al., 2015). Mitochondrial distribution is also correlated with the endoplasmic reticulum (Soltys \& Gupta, 1992). It is reported that mitochondria are remarkably mobile organelles. They can move in association with the microtubules from one

\footnotetext{
Manuscript received October 10, 2017, Received in revised form November 2, 2017, Accepted November 20, 2017

$\uparrow$ Corresponding Author: Gil Jung Kim, Department of Marine Molecular Bioscience, Gangneung-Wonju National University, 7 Jukheon-gil, Gangneung 25457, Republic of Korea. Tel: +82-33-640-2415, Fax: +82-33-640-2849, E-mail: gjkim@ gwnu.ac.kr

* These authors contributed equally to this work.

This is an Open Access article distributed under the terms of the Creative Commons Attribution Non-Commercial License (http:// creativecommons.org/licenses/by-nc/3.0) which permits unrestricted non-commercial use, distribution, and reproduction in any medium, provided the original work is properly cited.
} 
side to the other of the cells (Lawrence et al., 2016). In animal development, since distributions of mitochondria is closely related to cell differentiation, more mitochondria are transferred to the cells that use a lot of energy compared to other cells (Coffman \& Denegre, 2007; Dumollard et al., 2007). Usually, a large number of mitochondria are transferred to the muscle and neural precursor cells.

Ascidian embryos have become an important model for embryological studies, offering a simple example for mechanisms of differential segregation of cytoplasmic components. They have an invariant cleavage pattern with no cell migration or cell death up to the gastrula stage, and the developmental fate of each blastomere are invariant among individual embryos (Nishida, 1987). It is a well-known example that the differential segregation of mitochondria into muscle lineage cells occurs during ascidian embryogenesis (Zalokar \& Sardet, 1984; Fujiwara \& Satoh, 1990; Nishida, 1990). However, it is still unknown what signaling and molecular events control the polarized distribution of mitochondria. We have been interested in how the asymmetric distribution of mitochondria is achieved during ascidian embryogenesis. In this study, we show three-types of monoclonal antibodies that recognize mitochondria-rich cytoplasm in the ascidian embryo. The staining patterns of these antibodies are very similar to each other. Distribution pattern of mitochondria revealed by these antibodies coincided with that of $\mathrm{DiOC}_{2}$ experiment. These antibodies will be useful immunological probes for studying the polarized distribution of mitochondria in the ascidian embryo.

\section{MATERIALS AND METHODS}

\section{Embryos}

During the spawning season, Halocynthia roretzi adults were collected by fishermen in the vicinity of GangneungWonju National University, Gangneung, South Korea. Naturally spawned eggs were inseminated with a dilute suspension of non-self sperm and the fertilized eggs were raised in filtered sea water containing $50 \mu \mathrm{g} / \mathrm{mL}$ streptomycin at $13{ }^{\circ} \mathrm{C}$. At this temperature, tadpole larvae hatched about $35 \mathrm{hr}$ after fertilization.

\section{Production of monoclonal antibodies}

Monoclonal antibodies were produced as described by Fujiwara and Satoh (1990) and Kim and Nishida (1998). Embryos just before hatching were collected and homogenized in an ice-cold physiological saline solution. The homogenate was centrifuged at about $10,000 \times \mathrm{g}$ for $5 \mathrm{~min}$ at $4{ }^{\circ} \mathrm{C}$ to remove yolk granules. The supernatant was used as an immunogen. Female BALB/c mice were injected intraperitoneally with $0.5 \mathrm{~mL}$ supernatant. From the second immunization the mice were injected with the supernatant combined with Freund's incomplete adjuvant. The immunization was repeated five times. Three days after the last boost, mouse spleen cells were fused with PAI myeloma cells using $50 \%$ polyethylene glycol 4,000 Mw in serumfree Dulbecco's modified Eagle's medium (DMEM). The fused cells were separated into 96 multiwells and were cultured in DMEM containing hypoxanthine, aminopterin and thymidine. Each clone was assayed by indirect immunofluorescence as described below.

\section{Fixation and immunofluorescence staining}

Eggs and various stage embryos of Halocynthia were fixed for 10 minutes in $100 \%$ methanol at $-20^{\circ} \mathrm{C}$, and then in $100 \%$ ethanol for $10 \mathrm{~min}$ at $-20^{\circ} \mathrm{C}$. After dechorionation, the whole-mount specimens were washed with phosphatebuffered saline containing $0.05 \%$ Triton X-100 (PBSTr) and were stained with the hybridoma supernatant. Indirect immunofluorescence staining was carried out by standard methods using a TSA fluorescein system (PerkinElmer Life Sciences) according to the manufacturer's protocol. The monoclonal antibodies, Mito-1(5G10, 101), Mito-2 $(2 \mathrm{G} 3,32)$ and Mito-3 (3B8, 63), specifically recognizes mitochondria-rich cytoplasm in cells of Halocynthia em- 
bryos. 3, 3'-Diethyloxacarbocyanine lodide $\left(\mathrm{DiOC}_{2}\right)$ is a membrane potential fluorescent probe for vital staining of mitochondria and its validity in ascidian embryos has been demonstrated (Zaloker \& Sardet, 1984; Nishida, 1990). Dechorionated eggs and embryos were incubated in the filtered sea water that contained $0.5 \mu \mathrm{g} / \mathrm{mL} \mathrm{DiOC}_{2}$ (Thermo Fisher Scientific) for $1 \mathrm{hr}$ at $13{ }^{\circ} \mathrm{C}$. The specimens were rinsed several times and then transferred to new filtered sea water. Mitochondria were visualized by green emitted by the $\mathrm{DiOC}_{2}$ stain under a fluorescence microscope.

\section{RESULTS AND DISCUSSION}

To obtain specific immunological probes for studying mechanisms involved in the polarized distribution of mitochondria during ascidian embryogenesis, we have produced monoclonal antibodies against mitochondria of the embryos. We immunized mice with a homogenate of whole embryos, fused the mouse spleen cells to mouse myeloma cells to produce hybridoma cell lines. We screened individual hybridoma culture fluid samples by immunofluorescence staining. As described in Materials and Methods, more than 500 specimens were tested, from which we identified and cloned three hybridoma cell lines, 5G10 (Mito-1), 2G3 (Mito-2) and 3B8 (Mito-3), secreting antibodies that recognized mitochondria-rich cytoplasm in the eggs and embryos.

The Mito-1, Mito-2 and Mito-3 antibodies specifically recognized mitochondria-rich cytoplasm from eggs to tailbud embryos. The immunofluorescence results of these antiodies were very similar to each other (Fig. 1 and Fig. 2). At the early tailbud stage, each antibody stained cytoplasm like reticular structure in epidermis cells (Fig. 1B-1D). It seemed to be that the immunostaining signal was localized in the cytoplasm, except for nucleus. Similar staining pattern was observed in a vital staining of mitochondria with $\mathrm{DiOC}_{2}$, a fluorescent probe of mitochondria (Fig. 1A). It was reported that, in eukaryotic cells, mitochondria fuse

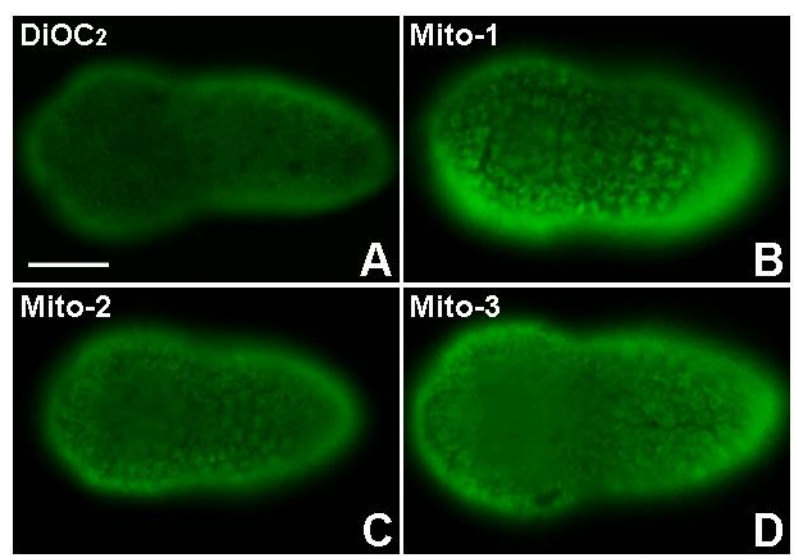

Fig. 1. Distribution of mitochondria in the early tailbud embryos of the ascidian, Halocynthia roretzi. (A) The embryo was stained with $\mathrm{DiOC}_{2}$, a fluorescent probe for vital staining of mitochondria. (B-D) Embryos were stained with three types of mitochondria-specific monoclonal antibodies, Mito-1, Mito-2 and Mito-3. In A, $\mathrm{DiOC}_{2}$ fluorescence is detected in cytoplasm of epidermis cells, except for nuclei. Similar staining pattern was observed in B-D. Scale bar, $100 \mu \mathrm{m}$.

to form elongated tubular networks around the nucleus (Egner et al., 2002; Friedman \& Nunnari, 2014). They are diffusely extended through the cytoplasm. Mitochondrial networks are dynamic, continually breaking apart by fission and fusing again. The features of mitochondria networks in cells were similarly observed in our experiments. Antigens that are recognized by the Mito-1, Mito- 2 and Mito-3 antibodies started to be detected from egg cytoplasm. The egg cytoplasm was also stained like networks with these antibodies (Fig. 2A). Mitochondria were dispersed over the cytoplasm, but they were localized more frequently in the cortical cytoplasm of eggs. A polarized distribution of mitochondria in the egg leads to the differential segregation of mitochondria between blastomeres. During cleavage stages, mitochondria are unevenly partitioned into blastomeres (Fig. 2B-2F). At the 8-cell stage, the Mito-1, Mito-2 and Mito-3 antibodies stained the posterior-vegetal B4.1 blastomeres, which mainly give rise 


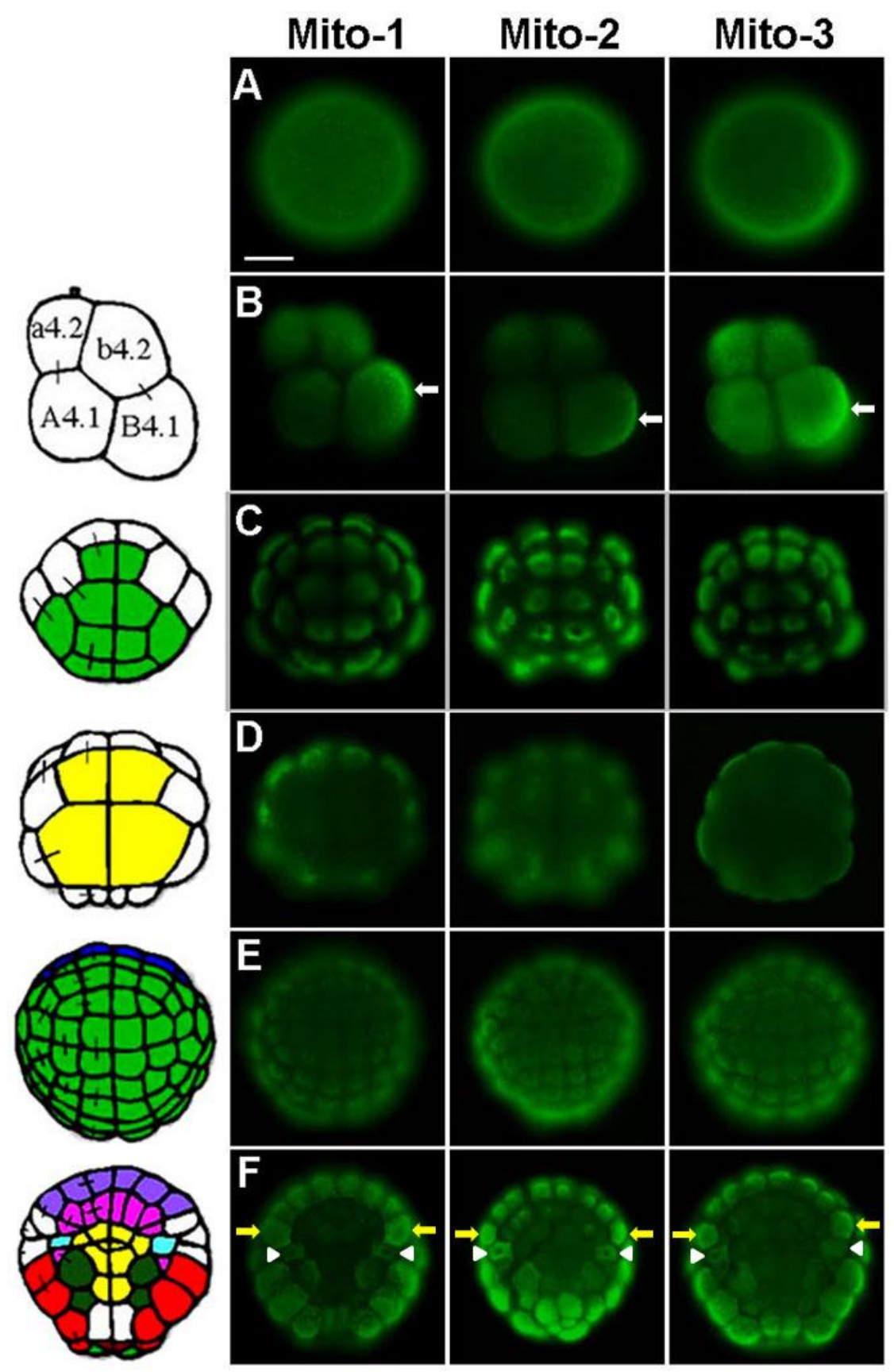

Fig. 2. Distribution of mitochondria visualized by immunofluorescence in the ascidian embryos. Immunostaining of eggs and various stage embryos with three types of mitochondria-specific monoclonal antibodies, Mito-1, Mito-2 and Mito-3. Schematic representations of embryos are shown on the left, with cell lineages are indicated by the particular colors (Original drawing by Dr Hiroki Nishida). Epidermis-lineage cells are colored green. Those for endoderm, the brain, nerve cord, notochord, TLCs (trunk lateral cells), mesenchyme and muscle are shown in yellow, blue, purple, pink, light blue, dark green and red, respectively. Bars connecting two blastomeres on the left side of the drawing embryos indicate sister cell relationship. (A) Fertilized eggs. (B) 8-cell stage embryos, lateral views. Anterior is to the left. White arrows indicate the posterior myoplasm-rich region of the B4.1 blastomeres. (C-D) 32- and (E-F) 110-cell stage embryos. Anterior is up. (C, E) Animal pole views. (D, F) Vegetal pole views. Yellow arrows indicate neural/secondary muscle-lineage blastomeres and white arrowheads represent TLCs. Scale bar, $100 \mu \mathrm{m}$. 
to muscle and mesenchyme cells, more strongly than other blastomeres. The immunofluorescence of mitochondria was particularly strong in the posterior myoplasm-rich region of the B4.1 blastomeres (Fig. 2B white arrows). This distribution pattern corresponds to that reported in the previous studies (Zalokar \& Sardet, 1984; Fujiwara \& Satoh, 1990; Roegiers et al., 1999). These studies showed that the B4.1 blastomeres contain the largest amount of mitochondria among all the blastomeres at the 8-cell stage. Mitochondria are equally distributed in the 2-cell stage embryo of the ascidian Phallusia, but the majority of mitochondria become segregated to the B4.1 blastomeres. In human embryos, differences in the mitochondrial distribution between blastomeres are obvious during the 2-cell and the 8cell stages (Van Blerkom, 2009). The asymmetric distribution can be traced back to polarized peri-nuclear aggregation at the one-cell stage. Similar asymmetry between blastomeres are also observed in mouse embryo (Acton et al., 2004). Thus, it is suggested that the asymmetric distribution of mitochondria initiated from a one-celled zygote is important for chordate development. It is likely that differential distribution of mitochondria is closely linked to cell fate choice.

The Mito-1, Mito-2 and Mito-3 antibodies showed that mitochondria are evenly distributed in the animal hemisphere blastomeres, which mainly form epidermis cells, at the 32-cell and the 110-cell stages (Fig. 2C, 2E). Immunostaining signals were localized mainly in the central cytoplasm, except for nuclei. In contrast, mitochondria appeared to be partitioned preferentially into blastomeres of the marginal zone, such as muscle (red) and nerve cord (purple) lineage, in the vegetal hemisphere (Fig. 2D, 2F). It appeared that endoderm (yellow) precursor cells which located in the central zone have been received the smallest amount of mitochondria. Mesenchyme (dark green) and notochord (pink) lineage blastomeres, located between muscle/nerve cord and endoderm, had a relatively small amount of mitochondria. At the 110-cell stage, distribution pattern of mitochondria revealed by these antibodies coincided with that of $\mathrm{DiOC}_{2}$ experiment (Fig. 2F and Fig. 3). $\mathrm{DiOC}_{2}$ fluorescence was detected strongly in the marginal zone blastomeres of the vegetal hemisphere more than in the central zone blastomeres. In addition, it was likely that endoderm blastomeres have the smallest amount of mitochondria. Therefore, it is suggested that these antibodies specifically recognize mitochondria of ascidian embryos, and they will be useful probes for studying mechanisms involved in asymmetric distribution of mitochondria in ascidian embryonic development. $\mathrm{DiOC}_{2}$ is a vital staining dye of mitochondria, whereas immunostaining with antibody requires fixing the specimens. Living specimens are advantageous to investigate successive changes in mitochondria distribution. The same specimen should be observed for a long time to examine the exact distribution of mitochondria. Immunostaining is more suitable for this. Thus, these

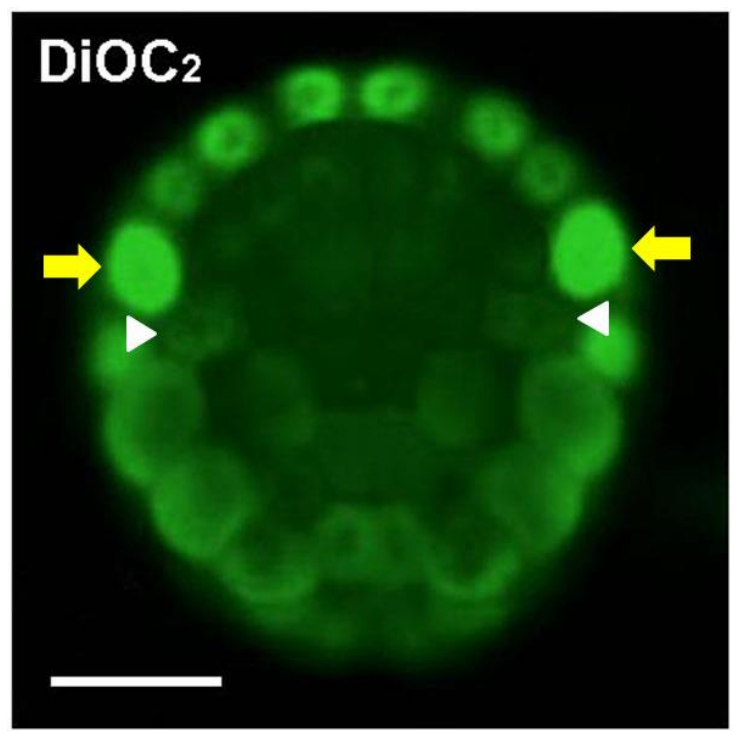

Fig. 3. Mitochondrial distribution in the vegetal hemisphere of the 110-cell stage embryos that was stained with $\mathrm{DiOC}_{2}$. Fluorescent signals of $\mathrm{DiOC}_{2}$ were detected more strongly in the marginal zone cells than in the central zone cells. Yellow arrows indicate neural/secondary muscle-lineage blastomeres and white arrowheads represent TLCs. This result coincided with that of fluorescence staining of antibodies. Scale bar, $100 \mu \mathrm{m}$. 
antibodies and $\mathrm{DiOC}_{2}$ are necessary to study the mechanisms involved in asymmetric distribution of mitochondria during ascidian embryogenesis.

In Fig. 2F and Fig. 3, yellow arrows indicate neural/ secondary muscle-lineage blastomeres and white arrowheads represent trunk lateral cells (TLCs). It is also clear that these cells had a large number of mitochondria. The TLCs are precursors of body-wall muscle and blood cells in adult organisms (Hirano \& Nishida, 1997). Considering that mitochondria are preferentially segregated into muscle and neural precursor cells during ascidian embryogenesis, these antibodies specifically recognize mitochondria. Such distribution pattern of mitochondria continued during gastrulation. At the early tailbud stage, muscle cells in tail possess a large amount of mitochondria (data not shown). At the 16-cell and the 32-cell stages, mitochondria which are segregated to one side of the vegetal hemisphere blastomeres transferred into one of the daughter cells (data not shown). In contrast, the polarized distribution of mitochondria did not observe in cytoplasm of the animal hemisphere blastomeres before cell division begins. Zalokar and Sardet (1984) have shown similar observations. However, the molecular events involved in the phenomenon are still unknown. It is probably important to isolate cDNA of the intracellular transport-related genes and to analyze roles of FGF signaling in the polarized distribution of mitochondria. FGF signaling promotes mesenchyme/notochord fates and suppresses muscle/nerve cord fate during ascidian embryogenesis (Kim et al., 2007). Thus, there is a possibility that FGF signaling involved in regulation of the differential segregation of mitochondria.

\section{ACKNOWLEDGEMENTS}

This research was supported by Basic Science Research Program through the Nation Research Foundation of Korea (NRF) funded by the Ministry of Education (2013R1A1A2 013658). Y.H.B and W.J.L received graduate fellowships from Gangneung-Wonju National University.

\section{REFERENCES}

Acton BM, Jurisicova A, Jurisica I, Casper RF (2004) Alterations in mitochondrial membrane potential during preimplantation stages of mouse and human embryo development. Mol Hum Reprod 10:23-32.

Alberts B, Bray D, Hopkin K, Johnson AD, Lewis J, Raff M, Roberts K, Walter P (2013) Essential Cell Biology. 4th ed. Garland Science, New York, pp 447-452.

Coffman JA, Denegre JM (2007) Mitochondria, redox signaling and axis specification in metazoan embryos. Dev Biol 308:266-280.

Dumollard R, Duchen M, Carroll J (2007) The role of mitochondrial function in the oocyte and embryo. Curr Top Dev Biol 77:21-49.

Egner A, Jakobs S, Hell SW (2002) Fast 100-nm resolution three-dimensional microscope reveals structural plasticity of mitochondria in live yeast. Proc Natl Acad Sci USA 99:3370-3375.

Friedman JR, Nunnari J (2014) Mitochondrial form and function. Nature 505:335-343.

Fujiwara S, Satoh N (1990) Pattern of segregation of mitochondria into muscle lineage cells during embryogenesis of the ascidian Halocynthia roretzi. Dev Growth Differ 32:531-539.

Hirano T, Nishida H (1997) Developmental fates of larval tissues after metamorphosis in ascidian Halocynthia roretzi. I. Origin of mesodermal tissues of the juvenile. Dev Biol 192:199-210.

Hoitzing H, Johnston IG, Jones NS (2015) What is the function of mitochondrial networks? A theoretical assessment of hypotheses and proposal for future research. BioEssays 37:687-700.

Kim GJ, Kumano G, Nishida H (2007) Cell fate polarization in ascidian mesenchyme/muscle precursors by directed FGF signaling and role for an additional ecto- 
dermal FGF antagonizing signal in notochord/nerve cord precursors. Development 134:1509-1518.

Kim GJ, Nishida H (1998) Monoclonal antibodies against differentiating mesenchyme cells in larvae of the ascidian Halocynthia roretzi. Zoolog Sci 15:553-559.

Lawrence EJ, Boucher E, Mandato CA (2016) Mitochondria-cytoskeleton associations in mammalian cytokinesis. Cell Div 11:3.

Nishida H (1987) Cell lineage analysis in ascidian embryos by intracellular injection of a tracer enzyme. III. Up to the tissue restricted stage. Dev Biol 121:526-541.

Nishida H (1990) Determinative mechanisms in secondary muscle lineages of ascidian embryos: development of muscle-specific features in isolated muscle progenitor cells. Development 108:559-568.

Roegiers F, Djediat C, Dumollard R, Rouvière C, Sardet C
(1999) Phases of cytoplasmic and cortical reorganizations of the ascidian zygote between fertilization and first division. Development 126:3101-3117.

Soltys BJ, Gupta RS (1992) Interrelationships of endoplasmic reticulum, mitochondria, intermediate filaments, and microtubules-a quadruple fluorescence labeling study. Biochem Cell Biol 70:1174-1186.

Tang HL, Lung HL, Wu KC, Le AP, Tang HM, Fung MC (2007) Vimentin supports mitochondrial morphology and organization. Biochemical J 410:141-146.

Van Blerkom J (2009) Mitochondria in early mammalian development. Semin Cell Dev Biol 20:354-364.

Zalokar M, Sardet C (1984) Tracing of cell lineage in embryonic development of Phallusia mammillata (Ascidia) by vital staining of mitochondria. Dev Biol 102: 195-205. 